\title{
Reply to comment on 'Density-functional theory of solid-to-solid isostructural transitions'
}

\author{
C N Likosł, Zs T Némethł and H Löwen§ \\ $\dagger$ Dipartimento di Fisica Teorica, Università di Trieste, Strada Costiera 11, I-34014 Grignano \\ (TS), Italy \\ $\ddagger$ University of Veszprém, Department of Physics, 8200 Veszprem, Hungary \\ $\S$ Sektion Physik der Ludwig-Maximilians-Universität München, Theresienstraße 37, D-80333 \\ München, Germany
}

Received 22 June 1995

Abstract. We reply to the preceding comment by Rascon and co-workers.

We reply to a comment by Rascón, Navascués and Mederos (RNM) [1] on a paper about the isosctructural fcc-fcc transitions discovered from simulations $[2,3]$ in systems characterized by short-range attractions [4]. The first point of RNM is that the approximation of the pair distribution function of the reference hard sphere (HS) system $g_{0}(r)$ (in the fluid phase) or $g_{0}\left(r, r^{\prime}\right)$ (in the solid phase) by a step function, which was employed in our paper [4], involves different degrees of approximation for the fluid and the solid phases. As a first consequence of this, RNM claim that the value of the width of the attractive part $\delta$ of the potential where the fcc-fcc coexistence ceases to exist, call it $\delta_{*}$, obtained in our theory is off by $41 \%$ compared to simulations. This was found by using the values $\delta_{*}=0.085$ from the theory and $\delta_{*}=0.06$ from simulations. This is not true. The correct values are $\delta_{*}=0.0824$ from the theory (as stated clearly in [4]) and $\delta_{*}=0.07$ from simulations (see [3] in the abstract), yielding an error of only $18 \%$, i.e. less than half the error that RNM report. Thus, the effect of this approximation is not as serious as RNM claim. In addition, RNM point out that the mean-field approximation for the fluid gives qualitatively incorrect results for the phase diagram when $\delta$ is further increased; in particular, it will fail to predict the liquid-gas phase coexistence of the Lennard-Jones system. However, it was made clear, both in the abstract and in the text that (i) we were proposing an approximate theory for systems interacting by means of short-range attraction only and (ii) our main goal was to study the novel and quite exciting phenomenon of the isostructural solid-to-solid transistion found in such systems from simulations, and not the liquid-gas separation. Our approach is a first attempt in this direction which, apart from giving quantitatively and qualitatively correct results, has the further advantage of being simple. Nowhere did we make a claim that our theory is applicable to long-range potentials. Moreover, we never attempted to examine the stabilities of all possible phases of the system (liquid, gas, dilute and dense fcc solids) for an arbitrary variation of the width of the attractive part of the potential. Thus, this criticism of RNM is irrelevant to our work.

In the remainder of their comment, RNM make some remarks on our suggestions for improving the theory beyond mean field. As a first way of achieving this improvement, we had suggested the possible inclusion of correlation effects through some approximation for the function $h_{0}\left(r, r^{\prime}\right)=g_{0}\left(r, r^{\prime}\right)-1$ for the solid (its liquid-state counterpart $h_{0}(r)$ 
being accurately known needs no approximations.) Here, RNM remark that (i) the function $h_{0}\left(r, r^{\prime}\right)$ is poorly known and (ii) that since $g_{0}\left(r, r^{\prime}\right)$ is relatively structureless, the inclusion of $h_{0}\left(r, r^{\prime}\right)$ will not necessarily produce a better estimate of the solid-solid critical temperature. We completely agree on count (i); it is difficult to obtain reliable information on the pair correlation function of the inhomogeneous system. Regarding (ii) we also agree that most likely $g_{0}\left(r, r^{\prime}\right)$ is devoid of the rich structure one observes at the fluid phase; however, at this point this is only an intuitive feeling and not a systematic result. Thus, the assumption that the inclusion of $h_{0}\left(r, r^{\prime}\right)$ will not affect significantly the critical temperature of the fccfcc transition (a very sensitive quantity!) is not based on any thorough investigation and therefore it does not constitute a strong criticism of our suggestions. RNM then point out that if one employs a different density-functional approximation for the reference (HS) system, then one obtains significantly different results for the critical temperature. This is correct, but also obvious and unrelated to our suggestions. It is clear that since most of the contribution to the free energy is contained on the reference part (in the sense of perturbation theory, in which our approach is based) a different calculation for the reference quantities will affect the result drastically. So, what we attempted to do in [4] was to use an accurate functional for the reference system (the modified weighted-density approximation [5]) and then we discussed ways to improve the approach beyond the zeroth order in perturbation theory.

As a second way of improving the theory, we suggested the following: since for shortrange attractions and for the range of temperatures which are interesting for the fcc-fcc coexistence (i.e. above the liquid-fcc-fcc triple temperature) the system possesses just one fluid phase, a thermodynamic mapping of the solid onto an effective liquid by means of one of the available nonperturbative density-functional approximations for the full interaction can in principle be made. In fact, this has now been done [6]. RNM have two objections here: first they say that for the range of $\delta$ which is interesting (small values) they have always found the usual van der Waals loop corresponding to the liquid-gas condensation. What they do not say is in which temperature range did they find this condensation to occur. The liquid-state theory employed in [6] shows precisely the same behaviour [7], but at temperatures much lower than the liquid-fcc-fcc triple temperature. Hence the mapping, which has to be performed at a range of temperatures where there exists just one fluid phase, is in principle possible. In fact, RNM say that the mapping '... will fail at least at low temperatures'. The point is that these temperatures are too low to be relevant, if one wants to examine the liquid-fcc-fcc part of the phase diagram. As a final remark, RNM state that the proposed strategy will only apply to a limited range of $\delta$-values. Here, we just reiterate that our whole approach, as well as our suggestions for improvements, were meant to be applicable to precisely small $\delta$-values, and we have stated that clearly in many places [8]. Thus, this remark is just a repetition of our statements, and not a correction. To conclude, we believe that most of the criticism of Rascón $e t a l$ is based on the misunderstanding that we had been proposing a theory which would apply to a much wider range of values for the model parameters (and the associated phenomena) than we had intended and clearly stated.

\section{Acknowledgments}

This work has been supported by the Human Capital and Mobility Programme of the Commission of the European Communities, contract No ERBCHBICT940940, and the Bundesministerium für Forschung und Technologie, contract No 03-WA3LMU. 


\section{References}

[1] Rascón C, Navascués G and Mederos L $1995 \mathrm{~J}$. Physs.: Condens. Matter 7

[2] Bolhuis P and Frenkel D 1994 Phys. Rev. Lett. 722211

[3] Bolhuis P, Hagen M and Frenkel D 1994 Phys. Rev. E 504880

[4] Likos C N, Németh Zs T and Löwen H 1994 J. Phys.: Condens. Matter 610965

[5] Denton A R and Ashcroft N W 1989 Phys. Rev. A 394701

[6] Likos C N and Senatore G 1995 J. Phys.: Condens. Matter 76797

[7] Nezbeda 11977 Czech. J, Phys. B 27247

[8] In fact, the liquid-state direct correlation function which was employed in [6] is based on expansions of quantities in powers of $\delta$, which immediately restricts its range of validity to small values of $\delta$ only (see [7] for details). 